\title{
Modeling elastic and photoassisted transport in organic molecular wires: length dependence and current-voltage characteristics
}

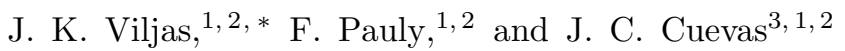 \\ ${ }^{1}$ Institut für Theoretische Festkörperphysik and DFG-Center for Functional Nanostructures, \\ Universität Karlsruhe, D-76128 Karlsruhe, Germany \\ ${ }^{2}$ Forschungszentrum Karlsruhe, Institut für Nanotechnologie, D-76021 Karlsruhe, Germany \\ ${ }^{3}$ Departamento de Física Teórica de la Materia Condensada, \\ Universidad Autónoma de Madrid, E-28049 Madrid, Spain
}

(Dated: July 17, 2008)

\begin{abstract}
Using a $\pi$-orbital tight-binding model, we study the elastic and photoassisted transport properties of metal-molecule-metal junctions based on oligophenylenes of varying lengths. The effect of monochromatic light is modeled with an ac voltage over the contact. We first show how the low-bias transmission function can be obtained analytically, using methods previously employed for simpler chain models. In particular, the decay coefficient of the off-resonant transmission is extracted by considering both a finite-length chain and infinitely extended polyphenylene. Based on these analytical results, we discuss the length dependence of the linear-response conductance, the thermopower, and the light-induced enhancement of the conductance in the limit of weak intensity and low frequency. In general the conductance-enhancement is calculated numerically as a function of the light frequency. Finally, we compute the current-voltage characteristics at finite dc voltages, and show that in the low-voltage regime, the effect of low-frequency light is to induce current steps with a voltage separation determined by twice the frequency. These effects are more pronounced for longer molecules. We study two different profiles for the dc and ac voltages, and it is found that the results are robust with respect to such variations. Although we concentrate here on the specific model of oligophenylenes, the results should be qualitatively similar for many other organic molecules with a large enough electronic gap.
\end{abstract}

PACS numbers: 73.50.Pz,85.65.+h,73.63.Rt

\section{INTRODUCTION}

The use of single-molecule electrical contacts for optoelectronic purposes such as light sources, light sensors, and photovoltaic devices is an exciting idea. Yet, due to the difficulties that light-matter interactions in nanoscale systems pose for theoretical and experimental investigations, the possibilities remain largely unexplored. Concerning experiments, it has been shown that light can be used to change the conformation of some molecules even when they are contacted to metallic electrodes, thus enabling light-controlled switching. $\underline{\underline{1}}$ Some evidence of photoassisted processes influencing the conductance of laser-irradiated metallic atomic contacts has also been obtained $\stackrel{2}{2}$ Theoretical investigations of light-related effects in molecular contacts

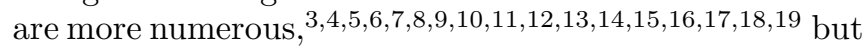
they are mostly based on highly simplified models, whose validity remains to be checked by more detailed calculations ${ }^{20,21}$ and experiments. However, for the description of the basic phenomenology, model approaches can be very fruitful, as they have been in studies of elastic transport in the past. Properties of linear single-orbital tight-binding (TB) chains, in particular, have been studied in detail, and to a large part analytically, $3,22,23,24,25,26,27,28,29,30,31,32$ In a step towards a more realistic description of the geometry, symmetries, and the electronic structure of particular molecules, empirical TB approaches such as the (extended) Hückel method have proved useful. $, 8,8,33,34,35$

Based on a combination of density-functional calculations and simple phenomenological considerations, we have recently described the photoconductance of metaloligophenylene-metal junctions. $\frac{5}{5}$ It was discussed how the linear-response conductance may increase by orders of magnitude in the presence of light. This effect can be seen as the result of a change in the character of the transport from off-resonant to resonant, due to the presence of photoassisted processes $\frac{5,7.8}{}$ Consequently, the decay of the conductance with molecular length is slowed down, possibly even making the conductance lengthindependent,$\underline{5,8}$

In this paper we apply a Hückel-type TB model of oligophenylene-based contacts 36 combined with Greenfunction methods $\frac{4}{*}$ to study the effects of monochromatic light on the dc current in metal-oligophenylene-metal contacts. Again we concentrate on the dependence of these effects on the length of the molecule. We begin with a detailed account of the elastic transport properties of the model, and show that the zero-bias transmission function can be obtained analytically, similarly to simpler chain models ${ }^{23,27}$ We demonstrate how information about the length dependence of the transmission function for a finite wire can be extracted from an infinitely extended polymer. Based on these analytical results, we discuss the length dependences of the conductance and the photoconductance for low-intensity and low-frequency light. While the conductance decays ex- 
ponentially with length, its relative enhancement due to light exhibits a quadratic behavior. Here we also briefly consider the thermopower, whose length dependence is linear. Next, we calculate numerically the zero-bias photoconductance as a function of the light frequency $\omega$, and find that the conductance-enhancement due to light is typically very large $\frac{3,5,8}{1}$ In particular, we show that the results of Ref. 5 are expected to be robust with respect to variations in the assumed voltage profiles. Finally, we describe how the step-like current-voltage ( $I$ $V)$ characteristics are modified by light. At high $\omega$ the most obvious effect is the overall increase in the lowbias current. At low $\omega$, additional current steps similar to those in microwave-irradiated superconducting tunnel junctions $\stackrel{37,38}{3}$ can be seen. Their separation, in our case of symmetric junctions, is roughly $2 \hbar \omega / e$.

TB models of the type we shall consider neglect various interaction effects (see Sec. $\mathrm{V}$ for a discussion), and thus cannot be expected to give quantitative predictions. However, the qualitative features of the results rely only on the tunneling-barrier character of the molecular contacts, which results from the fact that the Fermi energy of the metal lies in the gap between the highest-occupied and lowest-unoccupied molecular orbitals (HOMO and LUMO) of the molecule. Thus, these features should remain similar for junctions based on many other organic molecules exhibiting large HOMO-LUMO gaps. The light-induced effects, if verified experimentally, could be used for detecting light, or as an optical gate (or "third terminal") for purposes of switching.

The rest of the paper is organized as follows. In Sec. II we describe our theoretical approach, discuss the general properties of TB wire models, and introduce the Green-function method for the calculation of the elastic transmission function. Then, in Sec. III we calculate the transmission function of oligophenylene wires analytically. The decay coefficient for the off-resonant transmission is extracted also from infinitely extended polyphenylene. Following that, in Sec. IV we present our numerical results for the conductance, the thermopower, the photoconductance, and the $I-V$ characteristics. Finally, Sec. $\nabla$ ends with our conclusions and some discussion. Details on the calculation of the time-averaged current in the presence of light are deferred to the appendixes. In App. A simplified interpretation of the current formula is derived, and in App. B a brief account of the general method is given. Readers mainly interested in the discussion of the results for the physical observables can skip most of Secs. II and III and proceed to Sec. IV.

\section{THEORETICAL FRAMEWORK}

\section{A. Transport formalism}

Our treatment of the transport characteristics for the two-terminal molecular wires is based on Green's functions and the Landauer-Büttiker formalism, or its gen- eralizations. Assuming the transport to be fully elastic, the dc electrical current through a molecular wire can be described with

$$
I(V)=\frac{2 e}{h} \int d E \tau(E, V)\left[f_{L}(E)-f_{R}(E)\right] .
$$

Here $V$ is the dc voltage and $\tau(E, V)$ is the voltagedependent transmission function, while $f_{X}(E)=$ $1 /\left[\exp \left(\left(E-\mu_{X}\right) / k_{B} T_{X}\right)+1\right], \mu_{X}$, and $T_{X}$ are the Fermi function, the electrochemical potential, and the temperature of side $X=L, R$, respectively $\underline{39}$ The electrochemical potentials satisfy $\mathrm{eV}=\Delta \mu=\mu_{L}-\mu_{R}$, and we can choose them symmetrically as $\mu_{L}=E_{F}+e V / 2$ and $\mu_{R}=E_{F}-e V / 2$, where $E_{F}$ is the Fermi energy. For studies of dc current we always assume $T_{L}=T_{R}=0$. Of particular experimental interest is the linear-response conductance $G_{d c}=\partial I /\left.\partial V\right|_{V=0}$, given by the Landauer formula $G_{d c}=G_{0} \tau\left(E_{F}\right)$, where $G_{0}=2 e^{2} / h$ and $\tau(E)=\tau(E, V=0)$. In most junctions based on organic oligomers, the transport can be described as off-resonant tunneling. This results in the well-known exponential decay of $G_{d c}$ with the number $N$ of monomeric units in the molecule $\stackrel{40}{ }$ At finite voltages $V$, the current increases in a stepwise manner as molecular levels begin to enter the bias window between $\mu_{L}$ and $\mu_{R}$ (Ref. 24). We shall consider both of these phenomena below.

If a small temperature difference $\Delta T=T_{L}-T_{R}$ at an average temperature $T=\left(T_{L}+T_{R}\right) / 2$ is applied, heat currents and thermoelectric effects can arise $\underline{36,41,42}$ In an open-circuit situation, where the net current $I$ must vanish, a thermoelectric voltage $\Delta \mu / e$ is generated to balance the thermal diffusion of charge carriers. In the linear-response regime the proportionality constant $S=-(\Delta \mu / e \Delta T)_{I=0}$ is the Seebeck coefficient. We will briefly consider this quantity below as an example of an observable with a linear dependence on the molecular length $N$, but will not enter a more detailed discussion of thermoelectricity or heat transport.

The quantity we are most interested in is the dc current in the presence of monochromatic electromagnetic radiation, which we refer to as light independently of its source or frequency $\omega$. We model the light as an ac voltage with harmonic time-dependence $V(t)=V_{a c} \cos (\omega t)$ over the contact. The current averaged over one period of $V(t)$ can be written in the form ${ }^{3,4,43}$

$$
\begin{aligned}
I(V ; \alpha, \omega)= & \frac{2 e}{h} \sum_{k=-\infty}^{\infty} \int d E\left[\tau_{R L}^{(k)}(E, V ; \alpha, \omega)\right. \\
& \left.\times f_{L}(E)-\tau_{L R}^{(k)}(E, V ; \alpha, \omega) f_{R}(E)\right] .
\end{aligned}
$$

Here the transmission coefficient $\tau_{R L}^{(k)}(E)$, for example, describes photoassisted processes taking an electron from left $(L)$ to right $(R)$, under the absorption of a total of $k$ photons with energy $\hbar \omega$. The parameter $\alpha=e V_{a c} / \hbar \omega$ describes the strength of the ac drive $\underline{\underline{44}}$ It is determined by the intensity of the incident light and possible field-enhancement effects taking place in the metallic 


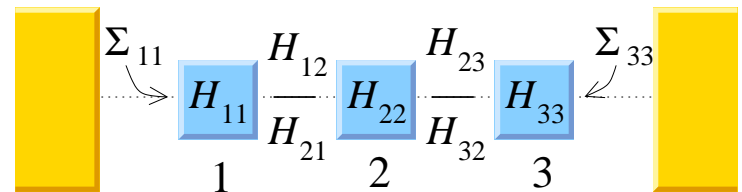

FIG. 1: (Color online) A finite block chain of length $N=3$ connected to electrodes at its two ends. This gives rise to self energies $\boldsymbol{\Sigma}_{11}$ and $\boldsymbol{\Sigma}_{N N}$ on the terminating blocks.

nanocontact $\underline{45}$ Again, in addition to the full $I-V$ characteristics, we study in more detail the case of linear response with respect to the dc bias, i.e., the photoconductance $G_{d c}(\alpha, \omega)=\partial I(V ; \alpha, \omega) /\left.\partial V\right|_{V=0}$. The arguments $\alpha$ and $\omega$ distinguish it from the conductance $G_{d c}$, although we sometimes omit $\alpha$ for notational simplicity. The calculation of the coefficients $\tau_{R L / L R}^{(k)}(E)$ is rather complicated in general,$\underline{4}$ and we defer comments on this procedure to App. B. Below we shall mostly refer to an approximate formula (see App. A) that can be expressed in terms of $\tau(E)$. This amounts to a treatment of the problem on the level of the Tien-Gordon approach $3.37,46$ The full Green-function formalism for systems involving ac driving is presented in Ref. 4 .

In noninteracting (non-self-consistent) models it is in general not clear how the voltage drop should be divided between the different regions of the wire, and the electrode-wire interfaces. A self-consistent treatment would be in order in particular for asymmetrically coupled molecules. We only concentrate on left-right symmetric junctions, where where both the dc and ac voltages $\left(V\right.$ and $\left.V_{a c}\right)$ are assumed to drop according to one of two different symmetrical profiles. The symmetry of the junctions excludes rectification effects, such as lightinduced dc photocurrents in the absence of a dc bias voltage $\stackrel{3.9 .45}{ }$ However, light can still have a strong influence on the transmission properties of the molecular contact, as will be discussed below. It will be shown that our conclusions are essentially independent of the assumed voltage profile.

\section{B. Wire models}

Below we will specialize to the case of a metaloligophenylene-metal junction. However, to make some general remarks, let us first consider a larger class of molecular wires that can be described as $N$ separate units forming a chain, where only the nearest neighbors are coupled (see Fig. 1). We only discuss the calculation of the elastic transmission function $\tau(E, V)$ here, as this will be the focus of our analytical considerations in Sec. III] From this quantity (at $V=0$ ), the various linear-response coefficients such as the conductance and the thermopower can be extracted. Furthermore, as already mentioned, it suffices for an approximate treatment of the amplitudes $\tau_{R L}^{(k)}(E)$ as well.
We assume a basis $\left|\chi_{p}^{(\alpha)}\right\rangle$ of local (atomic) orbitals, where $p=1, \ldots, N$ indexes the unit, while $\alpha=1, \ldots, M_{p}$ denotes the orbitals in each unit. 47 For simplicity, the basis is taken to be orthonormal, i.e. $\left\langle\chi_{p}^{(\alpha)} \mid \chi_{q}^{(\beta)}\right\rangle=$ $\delta_{\alpha \beta} \delta_{p q}$. The (time-independent) Hamiltonian $H_{p q}^{(\alpha, \beta)}=$ $\left\langle\chi_{p}^{(\alpha)}|\hat{H}| \chi_{q}^{(\beta)}\right\rangle$ of the wire is then of the block-tridiagonal form

$$
\boldsymbol{H}=\left(\begin{array}{ccccc}
\boldsymbol{H}_{11} & \boldsymbol{H}_{12} & & & \\
\boldsymbol{H}_{21} & \boldsymbol{H}_{22} & \boldsymbol{H}_{23} & & \\
& \ddots & \ddots & \ddots & \\
& & \boldsymbol{H}_{N-1, N-2} & \boldsymbol{H}_{N-1, N-1} & \boldsymbol{H}_{N-1, N} \\
& & & \boldsymbol{H}_{N, N-1} & \boldsymbol{H}_{N N}
\end{array}\right),
$$

where $\boldsymbol{H}_{p q}$ with $p, q=1, \ldots, N$ are $M_{p} \times M_{q}$ matrices. (The unindicated matrix elements are all zeros.)

In the non-equilibrium Green-function picture, the effect of coupling the chain to the electrodes is described in terms of "lead self energies" " ${ }^{48}$ We assume these to be located only on the terminal blocks of the chain, with components $\boldsymbol{\Sigma}_{11}$ and $\boldsymbol{\Sigma}_{N N}$. The inverse of the stationarystate retarded propagator for the coupled chain will then be of the form

$$
\boldsymbol{F}=\left(\begin{array}{ccccc}
\boldsymbol{F}_{11} & \boldsymbol{h}_{12} & & & \\
\boldsymbol{h}_{21} & \boldsymbol{h}_{22} & \boldsymbol{h}_{23} & & \\
& \ddots & \ddots & \ddots & \\
& & \boldsymbol{h}_{N-1, N-2} & \boldsymbol{h}_{N-1, N-1} & \boldsymbol{h}_{N-1, N} \\
& & & \boldsymbol{h}_{N, N-1} & \boldsymbol{F}_{N N}
\end{array}\right) .
$$

Here $\boldsymbol{h}_{p, p \pm 1}=-\boldsymbol{H}_{p, p \pm 1}, \boldsymbol{h}_{p p}=E_{+} \mathbf{1}_{p p}-\boldsymbol{H}_{p p}$, and $E_{+}=$ $E+i 0^{+}$, while $\boldsymbol{F}_{11}=\boldsymbol{h}_{11}-\boldsymbol{\Sigma}_{11}$ and $\boldsymbol{F}_{N N}=\boldsymbol{h}_{N N}-\boldsymbol{\Sigma}_{N N}$. Charge-transfer effects between the molecule and the metallic electrodes shift the molecular levels with respect to the Fermi energy $E_{F}$. In a TB model, these can be represented by shifting the diagonal elements of $\boldsymbol{H}$. Once a transport voltage $V$ is applied, further shifts are induced. In our model the voltage-induced shifts will be taken from simple model profiles, and the relative position of $E_{F}$ will be treated as a free parameter.

Effective numerical ways of calculating the propagator $\boldsymbol{G}=\boldsymbol{F}^{-1}$ for block-tridiagonal Hamiltonians exist $\underline{\underline{49}, 50}$ In Sec. III we shall be interested in a special case, where $\boldsymbol{H}_{p, p-1}=\boldsymbol{H}_{-1}, \boldsymbol{H}_{p, p+1}=\boldsymbol{H}_{1}$ and $\boldsymbol{H}_{p p}=\boldsymbol{H}_{0}$ with the same $\boldsymbol{H}_{1}=\boldsymbol{H}_{-1}^{T}$ and $\boldsymbol{H}_{0}$ (of dimension $M_{p}=M$ ) for all $p$, describing an oligomer of identical monomeric units. In such cases also analytical progress in calculating the current in Eq. (11) may be possible. Once the Green function $G$ is known, the transmission function is given by $\underline{48}$

$$
\tau(E, V)=\operatorname{Tr}\left[\boldsymbol{\Gamma}_{11} \boldsymbol{G}_{1 N} \boldsymbol{\Gamma}_{N N}\left(\boldsymbol{G}_{1 N}\right)^{\dagger}\right],
$$

where $\boldsymbol{\Gamma}_{11}=-2 \operatorname{Im} \boldsymbol{\Sigma}_{11}$ and $\boldsymbol{\Sigma}_{11}(E, V)=\boldsymbol{\Sigma}_{11}(E-e V / 2)$, for example.

Typically $E_{F}$ lies within the HOMO-LUMO gap, resulting in the exponential decay $\tau\left(E_{F}\right) \sim e^{-\beta\left(E_{F}\right) N}$ with 
$N$, characteristic of off-resonant transport. The decay coefficient $\beta\left(E_{F}\right)$ is actually independent of $\boldsymbol{\Sigma}_{11}$ and $\boldsymbol{\Sigma}_{N N}$. This can be seen by considering the Dyson equation $\boldsymbol{G}=\mathcal{G}+\mathcal{G} \boldsymbol{\Sigma} \boldsymbol{G}$, where $\boldsymbol{G}$ and $\mathcal{G}$ are the Green function of the coupled and uncoupled wires, respectively, and $\boldsymbol{\Sigma}$ is the matrix for the lead self-energies. Assuming that $\mathcal{G}_{1 N}$ decays exponentially with $N$, then

$$
\boldsymbol{G}_{1 N} \approx\left(\mathbf{1}-\mathcal{G}_{11} \boldsymbol{\Sigma}_{11}\right)^{-1} \mathcal{G}_{1 N}
$$

when $N \rightarrow \infty$, and therefore $\boldsymbol{G}_{1 N}$ decays with the same exponent. Thus, one can in principle obtain the decay exponent from the propagator of an isolated molecule, or even an infinitely extended polymer. In the next Section we demonstrate this by extracting the decay exponent of a finite oligophenylene junction from the propagator for polyphenylene. We note that in doing so, we neglect the practical difficulty of determining the correct relative position of $E_{F}$.

There are efficient numerical methods for computing the lead self-energies for different types of electrodes and various bonding situations between them and the wire. Typically, the methods are based on the calculation of surface Green's functions. $\frac{51}{=}$ Below we shall simply treat the self-energies as parameters.

\section{PHENYL-RING-BASED WIRES}

In this Section we discuss a special case of the type of wire model introduced above, describing an oligomer of phenyl rings coupled to each other via the para $(p)$ position ${ }^{36}$ The bias voltage $V$ is assumed to be zero. In the special case that we will consider, the inversion of Eq. (4) can then be done analytically with the subdeterminant method familiar from elementary linear algebra. $e^{23,24,27,32}$ Below, we first use this method for calculating the propagator of the finite-wire junction and derive the decay exponent $\beta(E)$ of the transmission function at off-resonant energies. After that we rederive the decay exponent by considering an infinitely extended polymer of phenyl rings.

\section{A. Oligo- $p$-phenylene junction}

Our model for the oligophenylene-based molecular junction is depicted in Fig. 2. Within a simple $\pi$-electron picture, the electronic structure of the oligophenylene molecule can be described with a nearest-neighbor TB model with two different hopping elements $-\gamma$ and $-\eta$ (Ref. 52). Here $-\gamma$ is for hopping within a phenyl ring, between the $p$ orbitals oriented perpendicular to the ring plane, while $-\eta$ describes hopping between adjacent rings. Due to the symmetry of the orbitals, the magnitude of $\eta$ depends on the angle $\varphi$ between the rings proportionally to $\cos \varphi$ (Ref. 53). We shall assume that $\eta=\gamma \cos \varphi$, and thus $|\eta| \leq \gamma$. In this way the natural energy scale of the model is set by $\gamma$ alone.

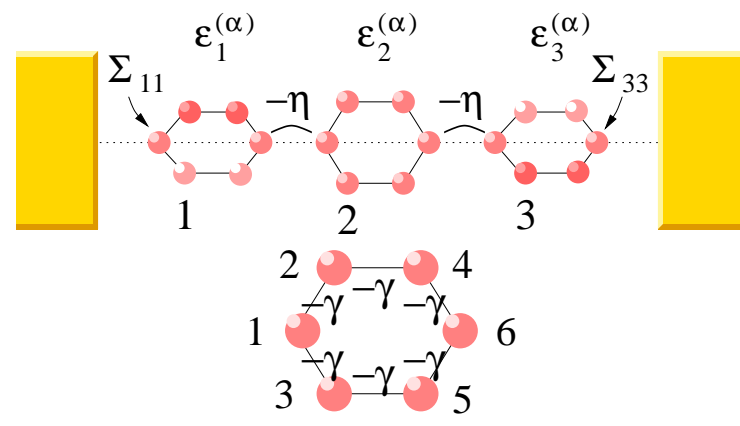

FIG. 2: (Color online) A finite chain of length $N=3$ connected to electrodes at its two ends. This gives rise to self energies $\boldsymbol{\Sigma}_{11}$ and $\boldsymbol{\Sigma}_{N N}$ on the end sites. The nearest-neighbor hoppings inside the ring $(-\gamma)$ and between the rings $(-\eta)$ are different. The lower part indicates also the numbering of the $M=6$ carbon atoms within a ring.

The ring-tilt angle $\varphi$ can be controlled to some extent using side groups. For example, two side groups bonded to adjacent phenyl rings can repel each other sterically, thus increasing the corresponding tilt angle $\stackrel{53,54}{ } \mathrm{In}$ fact, even the pure oligophenylenes in the uncharged state have $\varphi=30^{\circ}-40^{\circ}$ due to the repulsion of the hydrogen atoms $\frac{36.53}{3}$ However, side groups can introduce also "charging" or "doping" effects, which shift the molecular levels 55

For definiteness, we number the $M=6$ carbon atoms of a phenyl ring according to the lower part of Fig. 2. The corresponding orbitals appear in the basis in this order. Thus the blocks in Eq. (3) are

$$
\boldsymbol{H}_{q, q}=\left(\begin{array}{cccccc}
\epsilon_{q}^{(1)} & -\gamma & -\gamma & 0 & 0 & 0 \\
-\gamma & \epsilon_{q}^{(2)} & 0 & -\gamma & 0 & 0 \\
-\gamma & 0 & \epsilon_{q}^{(3)} & 0 & -\gamma & 0 \\
0 & -\gamma & 0 & \epsilon_{q}^{(4)} & 0 & -\gamma \\
0 & 0 & -\gamma & 0 & \epsilon_{q}^{(5)} & -\gamma \\
0 & 0 & 0 & -\gamma & -\gamma & \epsilon_{q}^{(6)}
\end{array}\right)
$$

for $q=1, \ldots, N$ and

$$
\boldsymbol{H}_{q, q-1}=\left(\begin{array}{cccccc}
0 & 0 & 0 & 0 & 0 & -\eta \\
0 & 0 & 0 & 0 & 0 & 0 \\
0 & 0 & 0 & 0 & 0 & 0 \\
0 & 0 & 0 & 0 & 0 & 0 \\
0 & 0 & 0 & 0 & 0 & 0 \\
0 & 0 & 0 & 0 & 0 & 0
\end{array}\right),
$$

with $\boldsymbol{H}_{q+1, q}=\left[\boldsymbol{H}_{q, q+1}\right]^{T}$. Here the onsite energies $\epsilon_{q}^{(\alpha)}$ may be shifted non-uniformly to describe effects of possible side-groups $\stackrel{36}{\underline{3}}$ For simplicity, we shall consider all phenyl rings to have a similar chemical environment, and thus all onsite energies are taken to be equal.

As a first step we note that, assuming $\epsilon_{q}^{(\alpha)}=\epsilon_{q}$ for all $\alpha$, the eigenvalues for the Hamiltonian $\boldsymbol{H}_{q q}$ of the isolated unit are $\epsilon_{q}-\gamma, \epsilon_{q}+\gamma, \epsilon_{q}-\gamma, \epsilon_{q}+\gamma, \epsilon_{q}-2 \gamma, \epsilon_{q}+2 \gamma$, 
while the corresponding orthonormalized eigenvectors are

$$
\begin{aligned}
& \frac{1}{\sqrt{4}}(0,-1,1,-1,1,0)^{T}, \frac{1}{\sqrt{4}}(0,1,-1,-1,1,0)^{T}, \\
& \frac{1}{\sqrt{12}}(-2,-1,-1,1,1,2)^{T}, \frac{1}{\sqrt{12}}(2,-1,-1,-1,-1,2)^{T}, \\
& \frac{1}{\sqrt{6}}(1,1,1,1,1,1)^{T}, \frac{1}{\sqrt{6}}(-1,1,1,-1,-1,1)^{T} .
\end{aligned}
$$

The first two of the eigenstates have zero weight on the ring-connecting carbon atoms 1 and 6 . Therefore, these eigenstates do not hybridize with the levels of the adjacent rings and consequently cannot take part in the transport. This will be seen explicitly in the derivation of the propagator. We note that these results can also be used to determine a realistic value for the hopping $\gamma$ from the HOMO-LUMO splitting of benzene. ${ }^{36}$

Below we shall only consider the analytically solvable case, where all onsite energies are set to the same value. We choose this value as our zero of energy: $\epsilon_{q}^{(\alpha)}=0$ for all $q=1, \ldots, N$ and $\alpha=1, \ldots, M$. Later on we shall relax this assumption in order to describe externally applied dc and ac voltage profiles. In the absence of such voltages, the inverse propagator [Eq. (4)] consists of the blocks $\boldsymbol{h}_{p, p}=\boldsymbol{h}_{0}, \boldsymbol{h}_{p, p-1}=\boldsymbol{h}_{-1}$, and $\boldsymbol{h}_{p, p+1}=\boldsymbol{h}_{1}$, where

$$
\begin{aligned}
\boldsymbol{h}_{0}= & \left(\begin{array}{ccccccc}
E_{+} & \gamma & \gamma & 0 & 0 & 0 \\
\gamma & E_{+} & 0 & \gamma & 0 & 0 \\
\gamma & 0 & E_{+} & 0 & \gamma & 0 \\
0 & \gamma & 0 & E_{+} & 0 & \gamma \\
0 & 0 & \gamma & 0 & E_{+} & \gamma \\
0 & 0 & 0 & \gamma & \gamma & E_{+}
\end{array}\right) \\
\boldsymbol{h}_{-1}= & \left(\begin{array}{llllll}
0 & 0 & 0 & 0 & 0 & \eta \\
0 & 0 & 0 & 0 & 0 & 0 \\
0 & 0 & 0 & 0 & 0 & 0 \\
0 & 0 & 0 & 0 & 0 & 0 \\
0 & 0 & 0 & 0 & 0 & 0 \\
0 & 0 & 0 & 0 & 0 & 0
\end{array}\right)
\end{aligned}
$$

and $\boldsymbol{h}_{1}=\left[\boldsymbol{h}_{-1}\right]^{T}$. The leads are assumed to couple only to the terminal carbon atoms, thus making the selfenergies $6 \times 6$ matrices of the form

$$
\boldsymbol{\Sigma}_{11}=\left(\begin{array}{cccc}
\Sigma_{L} & 0 & \cdots & 0 \\
0 & 0 & \cdots & 0 \\
\vdots & \vdots & \ddots & 0 \\
0 & 0 & 0 & 0
\end{array}\right), \quad \boldsymbol{\Sigma}_{N N}=\left(\begin{array}{cccc}
0 & 0 & 0 & 0 \\
0 & \ddots & \vdots & \vdots \\
0 & \cdots & 0 & 0 \\
0 & \cdots & 0 & \Sigma_{R}
\end{array}\right)
$$

We also define the symbol "tilde" ( ), which means the replacement of the first column of a matrix by $\eta$ followed by zeros. For example

$$
\tilde{\boldsymbol{h}}_{0}=\left(\begin{array}{cccccc}
\eta & \gamma & \gamma & 0 & 0 & 0 \\
0 & E_{+} & 0 & \gamma & 0 & 0 \\
0 & 0 & E_{+} & 0 & \gamma & 0 \\
0 & \gamma & 0 & E_{+} & 0 & \gamma \\
0 & 0 & \gamma & 0 & E_{+} & \gamma \\
0 & 0 & 0 & \gamma & \gamma & E_{+}
\end{array}\right)
$$

For the evaluation of Eq. (5), we only need the component $G_{1, M N}=\left[\boldsymbol{G}_{1 N}\right]_{1 M}$. Using the subdeterminants of $\boldsymbol{F}=\boldsymbol{G}^{-1}$, we have

$$
G_{1, M N}=\frac{(-1)^{M N+1} \operatorname{det}[\boldsymbol{F}(M N \mid 1)]}{\operatorname{det}[\boldsymbol{F}]} .
$$

Here $\boldsymbol{O}(i, \ldots, k \mid j, \ldots, l)$ is the submatrix of $\boldsymbol{O}$ obtained by removing the rows $i, \ldots, k$ and columns $j, \ldots, l$. We shall also denote by $L$ and $R$ the "leftmost" and "rightmost" row or column of a matrix. Thus, for example $\operatorname{det}[\boldsymbol{F}(M N \mid 1)]=\operatorname{det}[\boldsymbol{F}(R \mid L)]$

Let us first concentrate on the denominator of Eq. (13). It is easy to see that $\operatorname{det}[\boldsymbol{F}]$ can be written in terms of determinants related to the inverse Green function $\mathcal{F}=\mathcal{G}^{-1}$ of the uncoupled wire as follows ${ }^{23}$

$$
\begin{aligned}
\operatorname{det}[\boldsymbol{F}]=\operatorname{det}[\mathcal{F}] & -\Sigma_{L} \operatorname{det}[\mathcal{F}(L \mid L)]-\Sigma_{R} \operatorname{det}[\mathcal{F}(R \mid R)] \\
& +\Sigma_{L} \Sigma_{R} \operatorname{det}[\mathcal{F}(L, R \mid L, R)]
\end{aligned}
$$

Furthermore, due to the symmetry of the molecule, $\operatorname{det}[\mathcal{F}(R \mid R)]=\operatorname{det}[\mathcal{F}(L \mid L)]$. Thus we are left with calculating three types of determinants. It can be shown that, for $1<n<N$, all of them satisfy a recursion relation of the form

$$
\begin{aligned}
\left(\begin{array}{c}
D^{(n)} \\
\tilde{D}^{(n)}
\end{array}\right) & =\left(E_{+}^{2}-\gamma^{2}\right) \boldsymbol{Y}\left(\begin{array}{c}
D^{(n-1)} \\
\tilde{D}^{(n-1)}
\end{array}\right) \\
& =\left(E_{+}^{2}-\gamma^{2}\right)\left(\begin{array}{cc}
a & -c \\
c & b
\end{array}\right)\left(\begin{array}{l}
D^{(n-1)} \\
\tilde{D}^{(n-1)}
\end{array}\right) .
\end{aligned}
$$

For example, in the calculation of $\operatorname{det}[\mathcal{F}]$, we have $D^{(n)}=$ $\operatorname{det}\left[\mathcal{F}^{(n)}\right]$ and $\tilde{D}^{(n)}=\operatorname{det}\left[\tilde{\mathcal{F}}^{(n)}\right]$, where the additional superscript $(n)$ on the matrices denotes the number of the $M \times M$ diagonal blocks. The elements of the matrix $\boldsymbol{Y}$ are given by

$$
\begin{aligned}
a & =\left(E_{+}^{2}-\gamma^{2}\right)\left(E_{+}^{2}-4 \gamma^{2}\right) \\
b & =-\eta^{2}\left(E_{+}^{2}-\gamma^{2}\right) \\
c & =\eta E_{+}\left(E_{+}^{2}-3 \gamma^{2}\right) .
\end{aligned}
$$

Only the initial condition $(n=1)$ and the last step of the recursion $(n=N)$ will differ for the three determinants. The recursion relations can be solved by calculating $\boldsymbol{Y}^{n}$ explicitly, which can be done by diagonalizing $\boldsymbol{Y}$. The eigenvalues of $\boldsymbol{Y}$ are $\lambda_{1,2}=\left(a+b \mp \sqrt{(a-b)^{2}-4 c^{2}}\right) / 2$, while the (unnormalized) eigenvectors are

$$
\boldsymbol{v}_{1,2}=\left(\frac{a-b \mp \sqrt{(a-b)^{2}-4 c^{2}}}{2 c}, 1\right)^{T}
$$

Then, if $\boldsymbol{V}=\left(\boldsymbol{v}_{1}, \boldsymbol{v}_{2}\right)$ and $\boldsymbol{\Lambda}=\operatorname{diag}\left(\lambda_{1}, \lambda_{2}\right)$, we have $\boldsymbol{Y}^{n}=\boldsymbol{V} \boldsymbol{\Lambda}^{n} \boldsymbol{V}^{-1}$. The result is

$$
\boldsymbol{Y}^{n}=\left(\begin{array}{ll}
y_{11}^{(n)} & y_{12}^{(n)} \\
y_{21}^{(n)} & y_{22}^{(n)}
\end{array}\right)
$$


where the components are given by

$$
\begin{aligned}
& y_{11}^{(n)}=\frac{\left(\lambda_{1}^{n}-\lambda_{2}^{n}\right)(b-a)+\left(\lambda_{1}^{n}+\lambda_{2}^{n}\right) \sqrt{(a-b)^{2}-4 c^{2}}}{2 \sqrt{(a-b)^{2}-4 c^{2}}} \\
& y_{22}^{(n)}=\frac{\left(\lambda_{1}^{n}-\lambda_{2}^{n}\right)(a-b)+\left(\lambda_{1}^{n}+\lambda_{2}^{n}\right) \sqrt{(a-b)^{2}-4 c^{2}}}{2 \sqrt{(a-b)^{2}-4 c^{2}}} \\
& y_{12}^{(n)}=-y_{21}^{(n)}=\frac{c\left(\lambda_{1}^{n}-\lambda_{2}^{n}\right)}{\sqrt{(a-b)^{2}-4 c^{2}}} .
\end{aligned}
$$

Using these, we can now write explicit expressions for the three required determinants. For $\operatorname{det}[\mathcal{F}]$, the recursion can be started at $n=1$ with the initial conditions $D^{(0)}=$ 1 and $\tilde{D}^{(0)}=0$ and carried out up to $n=N$. The result is

$$
\operatorname{det}\left[\mathcal{F}^{(N)}\right]=\left(E_{+}^{2}-\gamma^{2}\right)^{N} y_{11}^{(N)} .
$$

The other two determinants require special initial and final steps, and the results are

$$
\begin{aligned}
\operatorname{det}\left[\mathcal{F}^{(N)}(L \mid L)\right]= & \left(E_{+}^{2}-\gamma^{2}\right)^{N} y_{21}^{(N)} / \eta \\
\operatorname{det}\left[\mathcal{F}^{(N)}(L, R \mid L, R)\right]= & \left(E_{+}^{2}-\gamma^{2}\right)^{N}\left[y_{21}^{(N-1)} c\right. \\
& \left.-y_{22}^{(N-1)} b\right] / \eta^{2} .
\end{aligned}
$$

Next, we consider the determinant in the numerator of Eq. (13), $\operatorname{det}\left[\boldsymbol{F}^{(N)}(R \mid L)\right]=\operatorname{det}\left[\mathcal{F}^{(N)}(R \mid L)\right]$. It can easily be shown that it satisfies the recursion relation

$$
\operatorname{det}\left[\mathcal{F}^{(N)}(R \mid L)\right]=2 \eta \gamma^{3}\left(E_{+}^{2}-\gamma^{2}\right) \operatorname{det}\left[\mathcal{F}^{(N-1)}(R \mid L)\right]
$$

and so

$$
\operatorname{det}\left[\mathcal{F}^{(N)}(R \mid L)\right]=2^{N}\left(\eta \gamma^{3}\right)^{N}\left(E_{+}^{2}-\gamma^{2}\right)^{N} / \eta .
$$

Now, the Green function of Eq. (13) can be written as

$$
\begin{aligned}
& G_{1, M N}= \\
& \frac{-\left(2 \eta \gamma^{3}\right)^{N} / \eta}{y_{11}^{(N)}+\Sigma_{L R} y_{21}^{(N)} / \eta+\Sigma_{L} \Sigma_{R}\left(y_{21}^{(N-1)} c-y_{22}^{(N-1)} b\right) / \eta^{2}},
\end{aligned}
$$

where we used the shorthand $\Sigma_{L R}=\Sigma_{L}+\Sigma_{R}$.

It is notable that the common $\left(E_{+}^{2}-\gamma^{2}\right)^{N}$ factors canceled out from the final propagator. These factors apparently correspond to the two eigenvectors of $\boldsymbol{h}_{0}$ [Eq. (9)] having zero weight on the ring-connecting atoms 1 and 6 . The cancellation is a manifestation of the physical fact that such localized states cannot contribute to the transport through the molecule. In the infinite polymer to be discussed below, these states appear as completely flat bands in the band structure.

To conclude this part, we point out that for $E$ inside the HOMO-LUMO gap [more precisely, when $(a-b)^{2}-$ $4 c^{2}>0$ ] the eigenvalues $\lambda_{1,2}$ are real-valued and the decay exponent of the transmission $\tau(E)$ for large $N$ is controlled by the one with a larger absolute value. Since
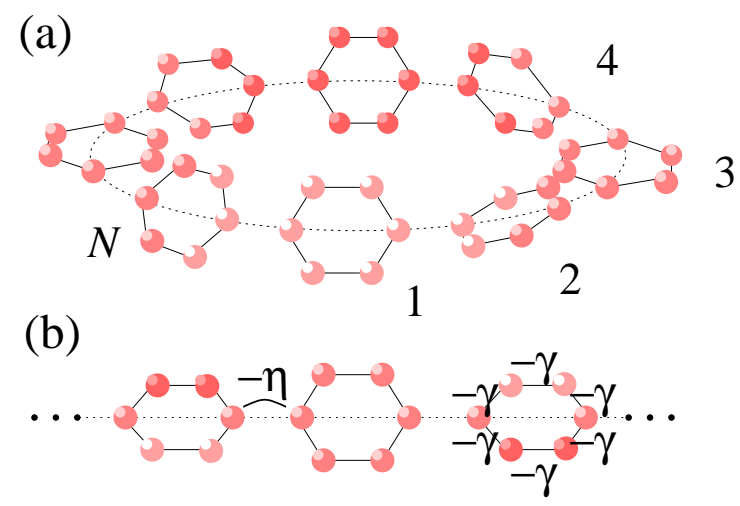

FIG. 3: (Color online) Phenyl-ring chains: (a) a periodic chain with $N$ units and (b) an infinite chain. Case (b) is obtained from (a) in the limit $N \rightarrow \infty$.

inside the gap $E \approx 0$, we find that $\lambda_{2}>\lambda_{1}>0$. Then, using Eq. (5) and omitting $N$-independent prefactors, the decay of the transmission for large $N$ follows the law

$$
\tau(E) \sim\left[\frac{\lambda_{2}(E)}{2 \eta \gamma^{3}}\right]^{-2 N}=e^{-2 N \ln \left[\lambda_{2}(E) /\left(2 \eta \gamma^{3}\right)\right]} .
$$

Thus the decay exponent is given by

$$
\beta(E)=2 \ln \left[\lambda_{2}(E) /\left(2 \eta \gamma^{3}\right)\right] .
$$

We note that for resonant energies, oscillatory dependence of $\tau(E)$ on $N$ can be expected, instead, and for limiting cases also power-law decay is possible $\underline{32}^{2}$ Next, we shall reproduce the result for the decay exponent by considering an infinitely extended polymer.

\section{B. Poly-p-phenylene}

For comparison with the "correct" evaluation of the propagator and the decay coefficient for a finite chain, let us consider the propagator for an infinitely extended polymer. To describe the polymer, we start from a finite chain with periodic boundary conditions. Neglecting curvature effects, the latter actually represents a ring-shaped oligomer, as depicted in Fig. 3(a).

Let us first consider the eigenstates of the periodic chain. The Hamiltonian $H_{p q}^{(\alpha, \beta)}=\left\langle\chi_{p}^{(\alpha)}|\hat{H}| \chi_{q}^{(\beta)}\right\rangle$ is of the general form

$$
\boldsymbol{H}=\left(\begin{array}{ccccc}
\boldsymbol{H}_{0} & \boldsymbol{H}_{1} & & & \boldsymbol{H}_{-1} \\
\boldsymbol{H}_{-1} & \boldsymbol{H}_{0} & \boldsymbol{H}_{1} & & \\
& \ddots & \ddots & \ddots & \\
& & \boldsymbol{H}_{-1} & \boldsymbol{H}_{0} & \boldsymbol{H}_{1} \\
\boldsymbol{H}_{1} & & & \boldsymbol{H}_{-1} & \boldsymbol{H}_{0}
\end{array}\right),
$$

where $\boldsymbol{H}_{0, \pm 1}$ are the $M \times M$ matrices $(M=6)$ of Eqs. (7) and (8), with $\epsilon_{q}^{(\alpha)}=0$. (Again, only nonzero elements 
are indicated.) The normalized eigenvectors $\boldsymbol{\psi}_{p}^{(n)}(k)$ satisfying

$$
\sum_{q} \boldsymbol{H}_{p q} \boldsymbol{\psi}_{q}^{(n)}(k)=E^{(n)}(k) \boldsymbol{\psi}_{p}^{(n)}(k)
$$

are of the Bloch form $\boldsymbol{\psi}_{q}^{(n)}(k)=e^{i k q d} \boldsymbol{\phi}^{(n)}(k) / \sqrt{N}$, where $\phi^{(n)}(k)$ are the normalized eigenvectors of

$$
\boldsymbol{H}(k)=e^{i k d} \boldsymbol{H}_{1}+\boldsymbol{H}_{0}+e^{-i k d} \boldsymbol{H}_{-1}
$$

with the eigenvalue $E^{(n)}(k)$, and $n=1, \ldots, M$. Due to the finiteness of the wire, the $k$ values are restricted to $k_{\mu}=2 \pi \mu / N d$, where $\mu$ is an integer and $d$ is the lattice constant (the length of a single phenyl-ring unit).

The spectral decomposition of the (retarded) propagator $\boldsymbol{g}(E)=\left(E_{+} \mathbf{1}-\boldsymbol{H}\right)^{-1}$ of the chain is of the form

$$
g_{p q}^{(\alpha, \beta)}(E)=\sum_{\mu, n} \frac{\left\langle\chi_{p}^{(\alpha)} \mid \psi^{(n)}\left(k_{\mu}\right)\right\rangle\left\langle\psi^{(n)}\left(k_{\mu}\right) \mid \chi_{q}^{(\beta)}\right\rangle}{E_{+}-E^{(n)}\left(k_{\mu}\right)},
$$

with the Bloch states

$$
\left|\psi^{(n)}\left(k_{\mu}\right)\right\rangle=\frac{1}{\sqrt{N}} \sum_{p=-\lceil N / 2\rceil+1}^{\lfloor N / 2\rfloor} e^{i k_{\mu} p d} \sum_{\alpha=1}^{M} \phi_{\alpha}^{(n)}\left(k_{\mu}\right)\left|\chi_{p}^{(\alpha)}\right\rangle .
$$

In the limit of large $N$ [Fig. [3) (b)], we can use $N^{-1} \sum_{\mu} \rightarrow$ $(d / 2 \pi) \int_{-\pi / d}^{\pi / d} d k$ to turn the summation into an integral over the first Brillouin zone. In this case, there are $M=6$ bands with energies

$$
\begin{aligned}
& E^{(1,2)}(k)= \pm \gamma \\
& E^{(3,4)}(k)= \pm \frac{1}{\sqrt{2}} \sqrt{\eta^{2}+5 \gamma^{2}-2 B(k)} \\
& E^{(5,6)}(k)= \pm \frac{1}{\sqrt{2}} \sqrt{\eta^{2}+5 \gamma^{2}+2 B(k)},
\end{aligned}
$$

where

$$
B(k)=\frac{1}{2} \sqrt{\left(\eta^{2}+3 \gamma^{2}\right)^{2}+16 \eta \gamma^{3} \cos (k d)} .
$$

Clearly we have the symmetries $E^{(1)}(k)=-E^{(2)}(k)$, $E^{(3)}(k)=-E^{(4)}(k)$, and $E^{(5)}(k)=-E^{(6)}(k)$. For $n=$ 1,2 the bands are completely flat, and the corresponding eigenvectors $\phi^{(1,2)}(k)$ are as in Eq. (9), i.e., independent of $k$ and completely localized on atoms $\alpha=2,3,4,5$. Thus for $p \neq q$, they do not contribute to the propagator in Eq. (30). For $n=3,4,5,6$, the vectors are very complicated, but they are not needed in the following.

To compare with the result of Sec. II A, we should now calculate, for example, the component $g_{p q}^{(1,6)}$. However, expecting the decay exponent to be independent of $\alpha$ and $\beta$, we consider the simpler case $\operatorname{Tr}\left[\boldsymbol{g}_{p q}\right]=\sum_{\alpha} g_{p q}^{(\alpha, \alpha)}$. Due to the orthonormality $\sum_{\alpha} \phi_{\alpha}^{(m)} \phi_{\alpha}^{(n) *}=\delta_{m n}$, the dependence on the vector components then drops out. Thus, for $p \neq q$

$$
\sum_{\alpha} g_{p q}^{(\alpha, \alpha)}=4 E A \frac{d}{2 \pi} \int_{-\pi / d}^{\pi / d} d k \frac{e^{i k d(p-q)}}{A^{2}-B^{2}(k)}
$$

where we defined

$$
A=E_{+}^{2}-\frac{1}{2}\left(\eta^{2}+5 \gamma^{2}\right)
$$

such that $E_{+}^{2}-\left[\epsilon^{(3,5)}(k)\right]^{2}=A \pm B(k)$. Defining now $z=$ $e^{i k d}$, the integral can be turned into a contour integral around the contour $|z|=1$

$$
\sum_{\alpha} g_{p q}^{(\alpha, \alpha)}=-\frac{2 E A}{2 \pi i \eta \gamma^{3}} \oint_{|z|=1} d z \frac{z^{p-q}}{\left(z-z_{+}\right)\left(z-z_{-}\right)},
$$

where the poles $z_{ \pm}$are determined from the equation $z^{2}-\left[4 A^{2}-\left(\eta^{2}+3 \gamma^{2}\right)^{2}\right]\left(8 \eta \gamma^{3}\right)^{-1} z+1=0$. They are given by

$$
z_{ \pm}=\frac{4 A^{2}-\left(\eta^{2}+3 \gamma^{2}\right)^{2}}{16 \eta \gamma^{3}} \pm \sqrt{\left[\frac{4 A^{2}-\left(\eta^{2}+3 \gamma^{2}\right)^{2}}{16 \eta \gamma^{3}}\right]^{2}-1}
$$

such that $z_{+}=1 / z_{-}$, and we choose the signs so that $z_{-}$is inside the contour $|z|=1$. In addition to this, assuming that $p<q$, there is a pole of order $q-p$ at $z=0$. The integral can then be evaluated using residue techniques, with the result

$$
\sum_{\alpha} g_{p q}^{(\alpha, \alpha)}=\frac{2 E A}{\eta \gamma^{3}} \frac{z_{+}^{p-q}}{z_{+}-z_{-}} .
$$

This leads to an exponential decay of the propagator with growing $q-p>0$, when $E$ is off-resonant (in which case $z_{ \pm}$are real-valued). Using this result, we can give an estimate for the decay of the transmission function [Eq. (5)] through a finite chain of length $N$ by replacing $G_{1, M N}$ with $\operatorname{Tr}\left[\boldsymbol{g}_{1 N}\right] / M$. This yields

$$
\tau(E) \sim\left[z_{+}(E)\right]^{-2 N}=e^{-2 N \ln \left[z_{+}(E)\right]},
$$

and thus the exponent

$$
\beta(E)=2 \ln \left[z_{+}(E)\right] .
$$

It can be checked that this result is, in fact, equal to the result [Eq. (26)] obtained for the finite chain.

It is thus seen explicitly that the decay coefficient of the off-resonant transmission does not in any way depend on the coupling of the molecule to the leads. It should be kept in mind, however, that the relative position of $E_{F}$ within the HOMO-LUMO gap depends on the electrodelead coupling and the charge transfer effects. This information is still needed for predicting the decay exponent $\beta\left(E_{F}\right)$ of the conductance.

The analytical results presented in this and the previous section can be used for understanding the behavior of the transmission function upon changes in the parameters. For example, it should be noted that when $\eta$ is made smaller, the band gap around $E \approx 0$ becomes larger, and at the same time the decay exponent $\beta(E)$ grows. In this way, the conductance of a molecular junction can be controlled, for example, by introducing side groups to control the tilt angles $\varphi$ between the phenyl rings $\stackrel{36,53}{ }$ 
(a)

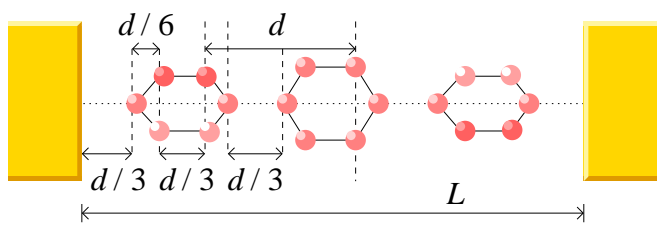

(b)

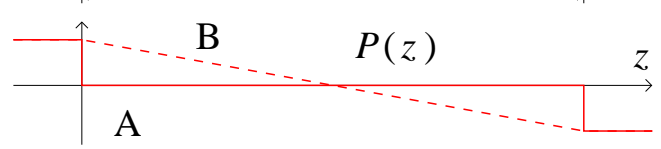

FIG. 4: (Color online) (a) The coordinates of the carbon atoms in the direction $z$ along the molecular wire. The left electrode is at $z=0$ and the length of a phenyl-ring unit is $d$. (b) Relative variation of the onsite energies for two different voltage profiles, $\mathrm{A}$ and $\mathrm{B}$. The profile function $P(z)$ describes how the harmonic voltage $V(t)=V+V_{a c} \cos (\omega t)$ is assumed to drop over the junction, the voltage at $z$ being given by $V(z, t)=V(t) P(z)$.

\section{PHYSICAL OBSERVABLES AND NUMERICAL RESULTS}

In this Section we present numerical results based on our model. Throughout, we employ the "wide-band" approximation for the lead self-energies, such that $\Sigma_{L}(E)=$ $-i \Gamma_{L} / 2$ and $\Sigma_{R}(E)=-i \Gamma_{R} / 2$, with energy-independent constants $\Gamma_{L, R}$. Furthermore we only consider the symmetric case $\Gamma_{L}=\Gamma_{R}=\Gamma$. First we briefly describe how we generalize the theory, as presented above, to take into account static and time-dependent voltage profiles. Then we concentrate on near-equilibrium (or "linearresponse") properties, using as examples the conductance, the thermopower, and the conductance enhancement due to light with low intensity and frequency. In this case, knowledge of the zero-bias transmission function calculated above is sufficient, and we can discuss the length dependence of the transport properties in a simple way. After that we consider the dc current in the presence of an ac driving field of more general amplitude and frequency, first concentrating on the case of infinitesimal dc bias, and finally on the $I-V$ characteristics.

\section{A. Voltage profiles}

When considering finite dc or ac biases within a nonselfconsistent TB model that cannot account for screening effects, one of the obvious problems is how to choose the voltage profile. Throughout the discussion, we shall refer to two possible choices, as depicted in Fig. 4. They are in some sense limiting cases, and the physically most reasonable choice should lie somewhere in between. Profile A assumes the external electric fields to be completely screened inside the molecule, such that the onsite energies are not modified, while B corresponds to the complete absence of such screening. In both cases, we can write the time-dependent onsite energies as $\epsilon_{p}^{(\alpha)}(t)=e V(t) P\left(z_{p}^{(\alpha)}\right)$, where $z_{p}^{(\alpha)}$ are the distances of the carbon atoms from the left metal surface, and $V(t)=V+V_{a c} \cos (\omega t)$. In case A, $P(z)=0$ inside the junction, while in case $\mathrm{B}$ $P(z)=(L-2 z) /(2 L)$, where $L=N d+d / 3$ is the distance between the two metal surfaces.

The profile B is more complicated, because the voltage ramp breaks the homogeneity of the wire. In this case the current must be calculated with the method outlined in App. B In the case of profile A, however, the $I-V$ characteristics can be calculated based on the knowledge of the zero-bias transmission function in the absence of light, $\tau(E)$. As discussed in App. A, the current is given by $3.46,56$

$$
\begin{aligned}
I(V ; \alpha, \omega)= & \frac{2 e}{h} \sum_{l=-\infty}^{\infty}\left[J_{l}\left(\frac{\alpha}{2}\right)\right]^{2} \int d E \tau(E+l \hbar \omega) \\
& \times\left[f_{L}(E)-f_{R}(E)\right] .
\end{aligned}
$$

The low-temperature zero-bias conductance then takes the particularly simple form $\stackrel{4,5}{ }$

$$
G_{d c}(\alpha, \omega)=G_{0} \sum_{l=-\infty}^{\infty}\left[J_{l}\left(\frac{\alpha}{2}\right)\right]^{2} \tau\left(E_{F}+l \hbar \omega\right) .
$$

Here $l$ indexes the number of absorbed or emitted photons, $J_{l}(x)$ is a Bessel function of the first kind (of order $l$ ), and $\alpha=e V_{a c} / \hbar \omega$ is the dimensionless parameter describing the strength of the ac drive. Note that $G_{d c}(\alpha, \omega=0)=G_{d c}(\alpha=0, \omega)=G_{0} \tau\left(E_{F}\right)=$ $G_{d c}$. Equation (41) may equally well be written in the form 37,57

$$
I(V ; \alpha, \omega)=\sum_{l=-\infty}^{\infty}\left[J_{l}\left(\frac{\alpha}{2}\right)\right]^{2} I_{0}(V+2 l \hbar \omega / e),
$$

where $I_{0}(V)$ is the $I-V$ characteristic in the absence of light [Eq. (1)]. Below, the results from these formulas are compared to the numerical results for profile B.

In Fig. 5 we plot the zero-bias transmission functions for wires with $N$ between 1 and 7 . Notice that the four energy bands numbered 3-6 in Eq. (32) are all visible, being separated by the HOMO-LUMO gap at $E / \gamma \approx 0$ and the additional gaps at $E / \gamma \approx \pm 1.7$. Here we use the parameters $\Gamma / \gamma=5.0, \varphi=40^{\circ}$ (i.e. $\eta / \gamma \approx 0.77$ ), and set the Fermi energy to $E_{F} / \gamma=-0.4$. These values are close to those used in Ref. 36, where they were extracted from a fit to results for gold-oligophenylene-gold contacts based on density-functional theory (DFT). We shall continue to use them everywhere below. A DFT calculation for the HOMO-LUMO splitting of benzene, together with the results preceding Eq. (9), yields the hopping $\gamma \approx 3$ $\mathrm{eV}$. The length of a phenyl-ring unit is approximately $d=0.44 \mathrm{~nm}$, and the largest ac electric fields $V_{a c} / L$ considered will be on the order of $10^{9} \mathrm{~V} / \mathrm{m}$. The photon energies $\hbar \omega$ will mainly be kept below the energy of the HOMO-LUMO gap of the oligophenylene. 


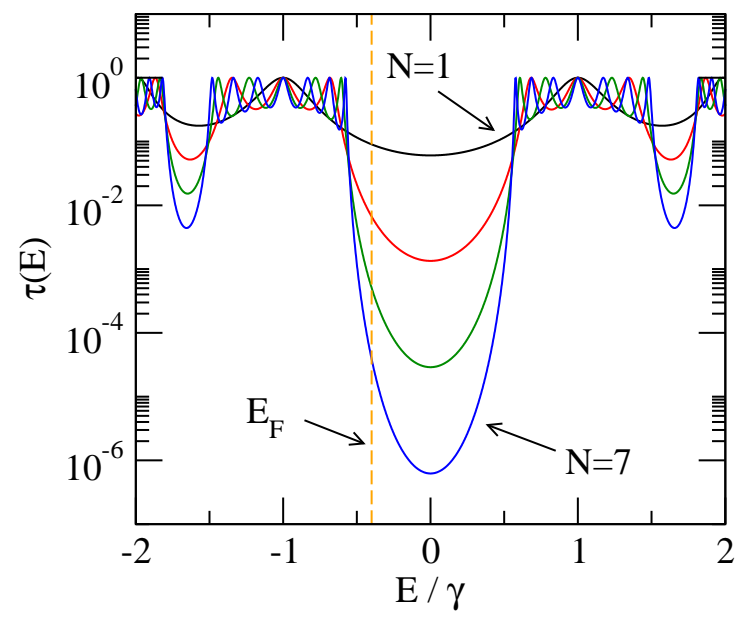

FIG. 5: (Color online) Transmission functions for the oligophenylene wires with lengths $N=1,3,5,7$. The parameters are $\Gamma / \gamma=0.5, \varphi=40^{\circ}, E_{F} / \gamma=-0.4$, as discussed in the text.
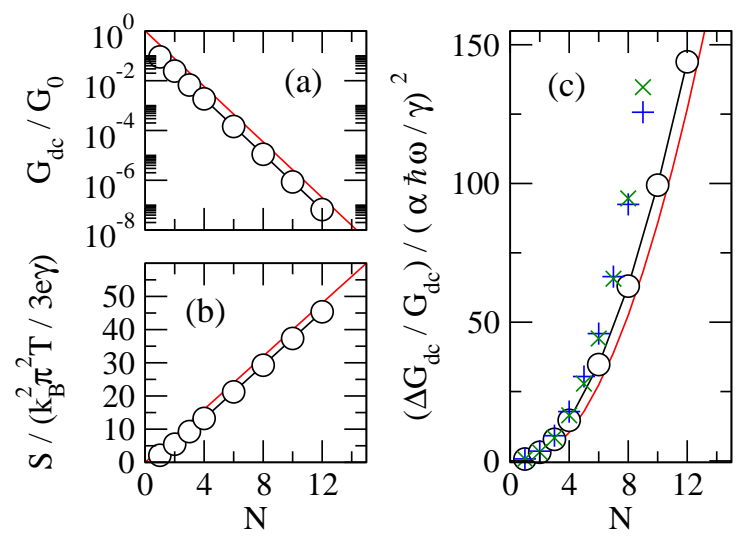

FIG. 6: (Color online) Dependence of observables on the number of units $N$ : (a) Conductance, (b) Seebeck coefficient, and (c) the light-induced relative conductance-enhancement. The circles correspond to values extracted from the $\tau(E)$ function [Fig. 5, using Eqs. (44), (45), and (46). The red lines correspond to the simple order-of magnitude estimates of Eqs. (47), (48), and (49), with the analytically calculated $\beta(E)$. In (c) the crosses $(\times$ for profile $\mathrm{A}$ and + for profile $\mathrm{B}$ ) show numerical results with the finite values $\alpha=0.5$ and $\hbar \omega / \gamma=0.05$ (see Sec. IVC).

\section{B. Near-equilibrium properties}

Let us start by illustrating the usefulness of the analytical results of Sec. III with a few examples. We concentrate on low temperatures and small deviations from equilibrium. In addition to the linear-response conductance

$$
G_{d c}=G_{0} \tau\left(E_{F}\right),
$$

we shall consider the thermopower, or Seebeck coefficient. At low enough temperature $T$, this is given in terms of the zero-bias transmission function $\tau(E)$ as $^{28,41,58,59}$

$$
S=-\frac{\pi^{2} k_{B}^{2} T}{3 e} \frac{\tau^{\prime}\left(E_{F}\right)}{\tau\left(E_{F}\right)},
$$

where prime denotes a derivative. Thus it measures the logarithmic first derivative of the transmission function at $E=E_{F}$. The sign of this quantity carries information about the location of the Fermi energy within the HOMO-LUMO gap of molecular junction $\stackrel{41}{\underline{4}}$ The third quantity we shall consider is the photoconductance. In the limit $\alpha \ll 1$ and $\hbar \omega / \gamma \ll 1$ we can expand $\tau(E)$ and the Bessel functions in Eq. (42) (see App. A) to leading order in these small quantities, yielding $G_{d c}(\omega)=$ $G_{0} \tau\left(E_{F}\right)+G_{0}(\alpha \hbar \omega)^{2} \tau^{\prime \prime}\left(E_{F}\right) / 16$. Defining then the lightinduced conductance correction $\Delta G_{d c}(\omega)=G_{d c}(\omega)-$ $G_{d c}(\omega=0)$, where $G_{d c}(\omega=0)=G_{d c}=G_{0} \tau\left(E_{F}\right)$, the relative correction becomes

$$
\frac{\Delta G_{d c}(\alpha, \omega)}{G_{d c}}=\frac{(\alpha \hbar \omega)^{2}}{16} \frac{\tau^{\prime \prime}\left(E_{F}\right)}{\tau\left(E_{F}\right)} .
$$

We thus see that this quantity gives experimental access to the second derivative of the transmission function at $E=E_{F}$. Note that in this approximation, which can be seen as an adiabatic or "classical" limit, $\frac{57}{,}$ the conductance correction depends only on the driving field through the ac amplitude $V_{a c}=\alpha \hbar \omega / e$.

As discussed above, it is reasonable to assume that for large enough $N$, the transmission function $\tau(E)$ satisfies the exponential decay law

$$
\tau(E) \sim C(E) e^{-\beta(E) N}
$$

at the off-resonant energies $E \approx E_{F}$. Let us furthermore assume that $C(E)$ is only weakly $E$-dependent. Then it is clear that the Seebeck coefficient will have the following simple linear dependence on $N$ (Refs. 28, 36):

$$
S \propto \tau^{\prime}\left(E_{F}\right) / \tau\left(E_{F}\right) \sim-\beta^{\prime}\left(E_{F}\right) N .
$$

In contrast, the light-induced conductance correction satisfies a quadratic law

$$
\begin{aligned}
\Delta G_{d c}(\omega) / G_{d c} & \propto \tau^{\prime \prime}\left(E_{F}\right) / \tau\left(E_{F}\right) \\
& \sim-\beta^{\prime \prime}\left(E_{F}\right) N+\left[\beta^{\prime}\left(E_{F}\right)\right]^{2} N^{2} .
\end{aligned}
$$

Deviations from these laws can follow from the energydependence of $C(E)$.

In Fig. 6] we demonstrate these length dependences within our model for the oligophenylene junctions. The circles connected by lines show the results based on the transmission functions of Fig. 5, using Eqs. (44), (45), and (46). The separate solid lines are the estimates of Eqs. (47), (48), and (49), based on the analytic result for $\beta(E)$. The result for $\Delta G_{d c}(\omega) / G_{d c}$ is furthermore compared with some example results for finite $\alpha$ and $\omega$, using $\alpha=0.5$ and $\hbar \omega / \gamma=0.05$ (see below). Although Eq. (46) was derived above by assuming the profile $\mathrm{A}$, the result appears to be rather well satisfied for profile B as well. 


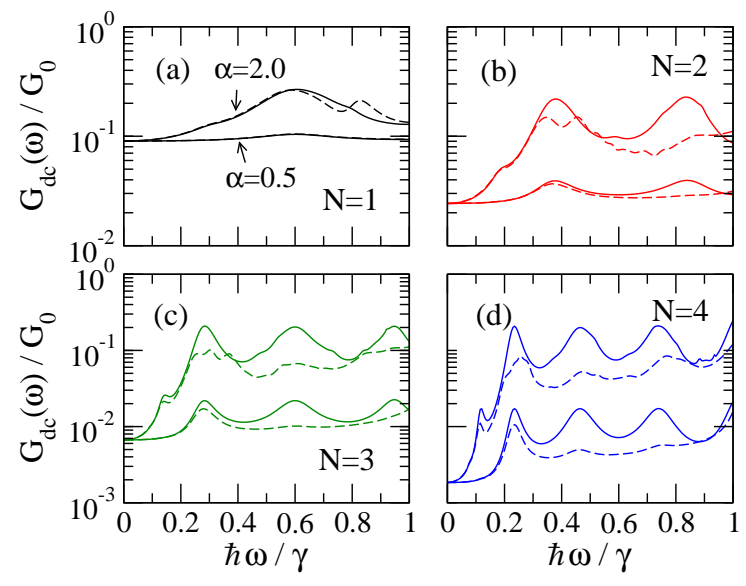

FIG. 7: (Color online) Zero-bias conductance for different driving frequencies $\omega$ and driving strengths $\alpha=e V_{a c} / \hbar \omega$. Panels (a)-(d) are for $N=1, \ldots, 4$. The solid lines correspond to profile A, and the dashed lines to profile B. The lower pair of curves is for $\alpha=0.5$, and the upper pair for $\alpha=2.0$.

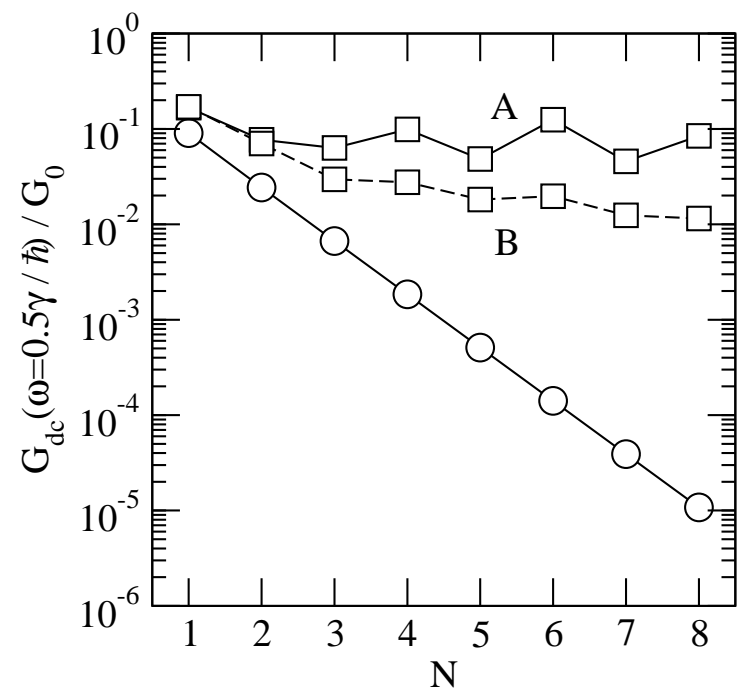

FIG. 8: (Color online) Dependence of the conductance on $N$. Circles represent the conductance in the absence of light, while the squares are for light with $\hbar \omega / \gamma=0.5$ and $\alpha=1.5$. The solid line is for profile A and the dashed line for profile B.

\section{Zero-bias conductance at finite drive frequencies and amplitudes}

Next we consider the zero-bias photoconductance $G_{d c}(\omega)$ for light whose frequencies and intensities are not restricted to the adiabatic limit. We have discussed this case previously, based on DFT results for goldoligophenylene-gold contacts. $\frac{5}{-}$ There, however, the analysis was based solely on the simple formula of Eq. (42). Here we show that those results are not expected to change in an essential way within a more refined theory, since the results of our TB model are not very different for the two voltage profiles A and B. This is seen in Fig. 7. where we show $G_{d c}(\omega)$ for $N=1, \ldots, 4$ as a function of $\omega$ for two values of $\alpha$, and for both profiles. The results for profile A again follow from Eq. (42), but the results for $\mathrm{B}$ require a more demanding numerical calculation (see App. B). In both cases the effect of light is to increase the conductance considerably. The physical reason is that the photoassisted processes, where electrons emit or absorb radiation quanta, brings the electrons to energies outside of the HOMO-LUMO gap, where the transmission probability is higher. This happens when $\hbar \omega$ exceeds the energy difference between the Fermi energy and the closest molecular orbital, in this case the HOMO. The main difference between the two profiles is that in case B, the sharp resonances at some frequencies are smeared out, and thus the light-induced conductance enhancement tends to be smaller. The increase can still be an order of magnitude or more.

The dependence of this effect on the length of the molecule is still illustrated in Fig. 8, where the conductances in the absence of light and in the presence of light with $\hbar \omega / \gamma=0.5$ and $\alpha=1.5$ are shown as a function of $N$. While the conductance in the absence of light has a strong exponential decay, in the presence of light this decay is much slower. For profile A the conductance actually oscillates periodically, while in the case of profile $\mathrm{B}$ the oscillations are superimposed on a background of slow exponential decay. In the DFT-based results 5 the oscillations were not present, or at least not visible for the cases $N=1, \ldots, 4$ considered there. Indeed, they are likely to be artifacts of the our TB model that neglects all other than $\pi$-orbital contributions, as well as uses the wide-band approximation.

The results of Fig. 8 can also be stated in terms of the relative conductance-enhancement $\Delta G_{d c}(\omega) / G_{d c}$. For large $\alpha$ and $\omega$, the increase of this quantity with $N$ is exponential for both profiles A and B. This should be contrasted with the quadratic behavior for small $\alpha$ and $\omega$ [Eq. (49)]. Thus, the fact that the results indicated by the crosses in Fig. 6 exceed the result of Eq. (46) is understandable.

\section{Current-voltage characteristics}

Finally we discuss the effects of light at finite voltages $V$. Let us first consider the properties of the $I-V$ characteristics in the absence of light. Examples are shown in Fig. 9(a) for the case $N=5$. They consist of consecutive steps, 60 which appear every time a new molecular level comes into the bias window between $\mu_{L}$ and $\mu_{R}$. These steps are seen as the peaks 1 and 2 in the differential conductance $d I / d V$ shown in Fig. 9(b). The first one occurs roughly at the voltage $V_{1}=2\left(E_{F}-E_{H O M O}\right) / e$, where $E_{H O M O}$ is the energy of the HOMO. The factor 2 arises from the symmetric division of the voltages with respect to the molecular energy levels. In the case of profile B, the currents tend to be smaller than for profile $\mathrm{A}$, but 

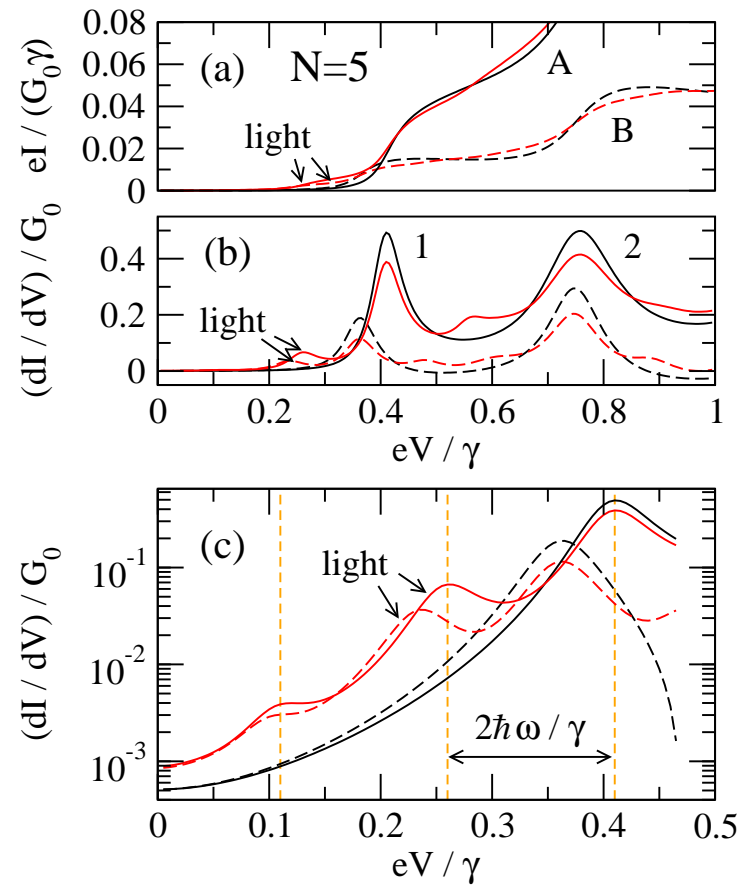

FIG. 9: (Color online) (a) $I-V$ characteristics $(N=5)$ with and without light for profiles A (solid lines) and B (dashed lines). Results in the presence of light with $\alpha=1.5$ and $\hbar \omega / \gamma=0.075$ are indicated with an arrow. (b) The corresponding differential conductances. (c) Same as (b), but concentrating on the low-bias regime and on a logarithmic scale. The vertical dashed lines indicate the approximate positions of the main peak and the light-induced side peaks. They are all separated by $2 \hbar \omega / e$ in voltage.

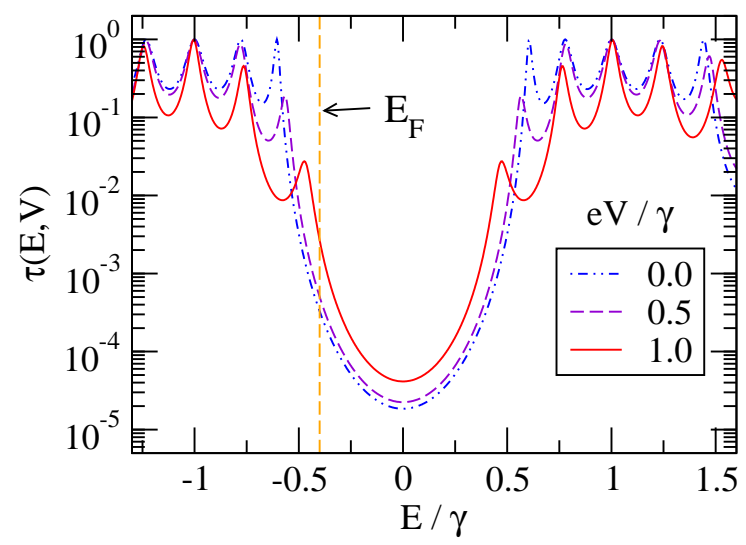

FIG. 10: (Color online) The voltage-dependent transmission function at three voltages for the wire with $N=5$ and profile B. For profile A the result is independent of voltage and equal to $\tau(E, V=0)$.

the current steps occur at roughly the same voltages. It should also be noticed that for profile $\mathrm{B}$, a small negative differential conductance is present following some of the steps. The origin of this is the localization of the molecular eigenstates due to the dc voltage ramp, which suppresses the transmission resonances $\stackrel{24}{\underline{24}}$ This can be seen
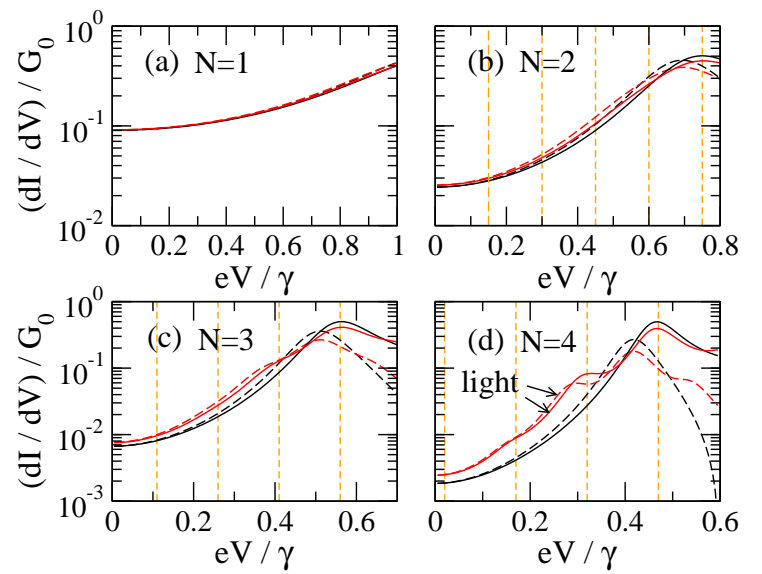

FIG. 11: (Color online) Same as Fig. 9(c) but for wires with $N=1, \ldots, 4$.

in the voltage-dependent transmission functions $\tau(E, V)$ in Fig. 10.

In the presence of light, the step structure of the $I-V$ curves is modified. For profile A, the results follow simply from Eq. (41) or (43), but for profile B a fully numerical treatment is again needed. In Fig. 9 the results for $\alpha=1.5$ and $\hbar \omega / \gamma=0.075$ are shown as the curves indicated with arrows. In Fig. 9(a) it is seen that the current for voltages below the steps is increased, and decreased above them. This removes the negative differential conductance present in the case of profile B. These changes are associated with the appearance of additional current steps. Here we concentrate only on the additional steps in the low-bias regime at voltages $V \lesssim V_{1}$, as the relative changes are largest there. Fig. 9(c) shows the differential conductance on a logarithmic scale in this voltage region. It can be seen that there are multiple extra peaks below the main peak, all of which are separated by voltages $2 \hbar \omega / e$ from each other. These peaks are "images" of the main peak at $V=V_{1}$, and are easily understood based on Eq. (43). For profile B all the peaks are moved to slightly smaller voltages and their spacing is reduced, since finite voltages tend to also suppress the transmission gap (see again Fig. (10). Notice that, in contrast to high dc biases [Fig. 9(a,b)], in the low-bias regime [Fig. 9(c)] the results depend only weakly on the choice of the voltage profile. Thus the predictions of the model appear to be robust. To observe the side steps, the radiation frequency should be large enough such that the steps are not "lost" under the broadening of the main steps. On the other hand, it should be small enough to have at least one step present. Thus, if the voltage broadening of the main step at $V=V_{1}$ is approximately $\Delta_{1} / e$, then we require $\Delta_{1} \lesssim \hbar \omega<E_{F}-E_{\text {HOMO }}$

Figure 11 additionally shows the low-bias differential conductances for $N=1, \ldots, 4$, with other parameters chosen as in Fig. 9(c). It is seen that the effects of light quickly become weaker, as the length of the molecule decreases. In the case $N=4$, small side peaks are still 
observed. Larger effects could be obtained by increasing the parameter $\alpha$.

Similar-looking additional steps are visible in the $I$ $V$ characteristics of an extended-Hückel model for xylyldithiol in Ref. 8. Despite the differences in magnitudes of parameters, and slight asymmetries in the geometries, it is likely that some of those steps have essentially the same origin as explained above. However, the most striking result in that reference was the overall order-of-magnitude increase in the current.

\section{CONCLUSIONS AND DISCUSSION}

In this paper we have studied a $\pi$-orbital tightbinding model to describe elastic and photoassisted transport through metal-molecule-metal contacts based on oligophenylenes. In contrast with simpler linear chain models that have previously been studied in great detail, our model describes a specific molecule, and its parameters can be directly associated with quantities obtainable from DFT simulations, for example. Models of this type can be of value in analyzing the results of more detailed $a b$-initio or DFT calculations,,$\frac{36}{=}$ and in making at least qualitative predictions in situations where such calculations would be prohibitively costly.

We first showed that at zero voltage bias the model can be studied analytically in a similar fashion as the simpler linear chain models. In particular, we derived an expression for the decay exponent of the off-resonant transmission function. We then discussed the length dependence of the dc conductance, the thermopower, and the relative light-induced conductance enhancement in the case of light with a low intensity $(\alpha)$ and low frequency $(\omega)$. The conductance enhancement was found to scale quadratically with length. For large $\alpha$ and $\omega$, the relative enhancement increases exponentially with length. Finally it was shown, by numerical calculations, that the current-voltage characteristics are modified in the presence of light by the appearance of side steps with a voltage spacing $2 \hbar \omega / e$. We demonstrated that the predictions of the model are robust with respect to variations in the assumed voltage profiles. This provides further support for our previous results on the photoconductance $\frac{\sqrt{5}}{}$

In our work, only symmetrical junctions with symmetrical voltage profiles were studied. Asymmetries can modify our results through the introduction of rectifica-

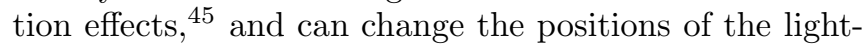
induced current steps. The experimental observation of additional steps with a spacing related to the frequency of the light would nevertheless provide more compelling evidence for the presence of photoassisted transport than a conductance enhancement alone. The latter can also have other causes $\underline{\underline{2}}$

We note that the light-induced current steps are similar to the steps observed in current-voltage characteristics of microwave-irradiated superconducting tunnel junctions, where they result from photoassisted quasiparticle tunneling, 37,61 In that case, the main difference is that the energy gap necessary for the effect is located in the macroscopic electrodes, while the transmission through the tunnel barrier depends only weakly on energy and voltage. As a result, the current steps have a voltage spacing of precisely $\hbar \omega / e$. These effects are exploited in the detection of microwaves in radioastronomy. 57 Similarly, one may imagine properly engineered molecular contacts as detectors of light in the infrared or visible frequency range.

In terms of our model, to increase the chances of observing the light-induced current steps, the aim should be to minimize the broadening $\Delta_{1} / e$ of the first main current step at voltage $V_{1}$, and to maximize $\alpha$. Also, a wire with a large enough $V_{1}$ should be used. The broadening $\Delta_{1}$ is related to the sharpness of the transmission resonances, and thus to the length of the molecule and its coupling to the electrodes, described by $\Gamma$. A decrease of $\Gamma$, however, increases the importance of Coulomb correlations. Their effect on photoassisted transport has recently been discussed within simple models. $\stackrel{15,62}{1 n}$ Increase of $\alpha$ through the light intensity, in turn, increases the heating of the electrodes ${ }^{2}$ and the excitation of local molecular vibrations $\underline{\underline{51}}$ These may affect the geometry through thermal expansion ${ }^{45}$ and structural deformations, but will also give rise to an incoherent component to the current $\underline{63}$ At high enough photon energies, also the direct excitation of electrons on the molecule may become important. The relaxation of such excitations due to various mechanisms (creation of electron-hole pairs in the electrodes, spontaneous light emission) should thus also be considered $\stackrel{9}{?}$ Also conformational changes of the molecule are possible $\underline{\underline{1}}$ Finally, a proper treatment of screening effects on the molecule and in the electrodes, the excitation of plasmons, and their role in the field enhancement $\$ \frac{45}{}$ are other issues that should be studied in more detail.

Of course, for the investigation of most of these issues, noninteracting models of the type presented above are not sufficient. Strong time-dependent electric fields may have effects that can only be captured by selfconsistent theories taking properly into account the electron correlations due to Coulomb interactions. These interactions may influence the electronic structure in a way that would, at least, require the parameters of our model to be readjusted in the presence of the light. Even the geometry of the junction can become unstable, and so it should in principle be optimized with the light-induced effects included. Time-dependent densityfunctional theory is showing some promise for the treatment of such problems ${ }^{20,21}$ In addition to DFT, also more advanced computational schemes are being developed to handle correlation effects ${ }^{64,65}$ A systematic investigation of the optical response of metal-moleculemetal contacts, and thus the testing of the predictions of the simple models,,$\frac{3,5,6,7,8,9}{2}$ remains an important goal for future research. 


\section{Acknowledgments}

This work was financially supported by the Helmholtz Gemeinschaft (Contract No. VH-NG-029), by the DFG within the Center for Functional Nanostructures, and by the EU network BIMORE (Grant No. MRTN-CT-2006035859). F. Pauly acknowledges the funding of a Young Investigator Group at KIT.

\section{APPENDIX A: SIMPLIFIED FORMULA FOR THE TIME-AVERAGED CURRENT}

Consider the expression Eq. (2) for the time-averaged (or dc) current. The coefficient $\tau_{R L}^{(k)}(E)$, for example, is the sum of the transmission probabilities of all transport channels taking the electron from energy $E$ on the left to energy $E+k \hbar \omega$ on the right. That is, for $k>0$ $(k<0)$ it describes electron transmission under the absorption (emission) of $k$ photons. Assuming the wideband approximation and the voltage profile A, Eq. (2) can be written in the more transparent forms of Eqs. (41) and (43). This can be demonstrated rigorously using the equations of App. B. but it is instructive to consider the following simpler derivation. The idea is the same as in the "independent channel approximation" of Ref. 7 .

For now, we allow the ac voltage drops at the $L$ and $R$ lead-molecule interfaces to be asymmetrical. Thus we define the quantities $\alpha_{L}$ and $\alpha_{R}$, satisfying $\alpha=\alpha_{L}-$ $\alpha_{R}$. Since for profile A there is no voltage drop on the molecule, electronic transitions only occur at the leadmolecule interfaces. Thus the transmission coefficients $\tau_{R L}^{(k)}(E)$ are given by

$$
\tau_{R L}^{(k)}(E)=\sum_{l=-\infty}^{\infty}\left[J_{l-k}\left(\alpha_{R}\right)\right]^{2} \tau(E+l \hbar \omega)\left[J_{l}\left(\alpha_{L}\right)\right]^{2}
$$

where $\left[J_{l}\left(\alpha_{L}\right)\right]^{2}$ is the probability for absorbing (emitting) $l$ photons on the left interface and $\left[J_{l-k}\left(\alpha_{R}\right)\right]^{2}$ the probability for emitting (absorbing) $l-k$ photons on the right interface. The propagation between the interfaces occurs elastically at the intermediate energy $E+l \hbar \omega$, according to the transmission function $\tau(E)$. A similar expression holds for $\tau_{L R}^{(k)}(E)$. Using these and the sum formula $\sum_{k=-\infty}^{\infty}\left[J_{k}(x)\right]^{2}=1$, Eq. (2) leads to

$$
\begin{aligned}
I(V ; \alpha, \omega) & =\frac{2 e}{h} \sum_{l=-\infty}^{\infty} \int d E \tau(E+l \hbar \omega) \\
& \times\left\{\left[J_{l}\left(\alpha_{L}\right)\right]^{2} f_{L}(E)-\left[J_{l}\left(\alpha_{R}\right)\right]^{2} f_{R}(E)\right\}
\end{aligned}
$$

Equation (41) follows by setting $\alpha_{L}=\alpha / 2$ and $\alpha_{R}=$ $-\alpha / 2$, and the equivalent form of Eq. (43) follows by changing summation indices and integration variables. Similarly, other suggestive forms may be derived. $3,46,56$
For $x \ll 1$ and $l>0$ one may expand $J_{ \pm l}(x) \approx$ $( \pm x / 2)^{l} / l !-( \pm x / 2)^{l+2} /(l+1)$ !. This can be used in the limit $\alpha \ll 1, \hbar \omega / \gamma \ll 1$ discussed in the text.

\section{APPENDIX B: GREEN'S-FUNCTION METHOD FOR THE TIME-AVERAGED CURRENT}

Here we outline the Green-function method 4,7,66 used for obtaining the results for voltage profile B. Consider again the dc current of Eq. (2). In the case of a harmonic driving field, it is reasonable to assume the existence of time-reversal invariance, in which case we have the symmetry ${ }^{3}$

$$
\tau_{L R}^{(k)}(E)=\tau_{R L}^{(-k)}(E+k \hbar \omega) .
$$

The current expression of Eq. (8) in Ref. 4 was derived under this assumption, and that result can be brought into the form of Eq. (2). Using the notation of that reference,,$\frac{47}{4}$ the coefficients can be written

$$
\begin{aligned}
\tau_{R L}^{(k)}(E) & =\operatorname{Tr}_{\omega}\left[\hat{G}(E) \hat{\Gamma}_{R}^{(k)}(E) \hat{G}^{\dagger}(E) \hat{\Gamma}_{L}^{(0)}(E)\right] \\
\tau_{L R}^{(k)}(E) & =\operatorname{Tr}_{\omega}\left[\hat{G}(E) \hat{\Gamma}_{L}^{(k)}(E) \hat{G}^{\dagger}(E) \hat{\Gamma}_{R}^{(0)}(E)\right],
\end{aligned}
$$

where the hats denote the extended "harmonic" matrices ${ }^{67}$ and $\operatorname{Tr}_{\omega}$ a trace over them. In particular, $\hat{G}$ is the matrix for the retarded propagator

$$
\hat{G}(E)=\left[(\hat{E}-\boldsymbol{H} \hat{1})-\hat{W}-\hat{\Sigma}_{L}(E)-\hat{\Sigma}_{R}(E)\right]^{-1},
$$

where $\boldsymbol{H}$ is the Hamiltonian of the wire in the absence of voltage profiles. The matrix $\hat{E}$ is defined by $[\hat{E}]_{m, n}=(E+m \hbar \omega) \delta_{m, n} \mathbf{1}$, where $m$ and $n$ are the harmonic indices. Using the wide-band approximation for the electrodes, the matrices $\hat{\Sigma}_{X}$ and $\hat{\Gamma}_{X}^{(l)}$ are given by

$$
\begin{aligned}
& {\left[\hat{\Sigma}_{X}\right]_{m, n}(E)=\delta_{m, n} \boldsymbol{\Sigma}_{X}} \\
& {\left[\hat{\Gamma}_{X}^{(l)}\right]_{m, n}(E)=J_{m-l}\left(\alpha_{X}\right) J_{n-l}\left(\alpha_{X}\right) \boldsymbol{\Gamma}_{X},}
\end{aligned}
$$

with $X=L, R$ and $\alpha_{L, R}= \pm \alpha / 2$. Here $\boldsymbol{\Sigma}_{X}$ is the selfenergy matrix of lead $X$ (extended to the size of $\boldsymbol{H}$ ), and $\boldsymbol{\Gamma}_{X}=-2 \operatorname{Im} \boldsymbol{\Sigma}_{X}$. The matrix $\hat{W}$ includes the effect of the profiles for the voltage $V(t)=V+V_{a c} \cos (\omega t)$. If $\boldsymbol{W}(t)=$ $\boldsymbol{W}_{d c}+\boldsymbol{W}_{a c} \cos (\omega t)$ is a diagonal matrix consisting of the onsite energies $\epsilon_{p}^{(\alpha)}(t)$, then

$$
[\hat{W}]_{m, n}=\boldsymbol{W}_{d c} \delta_{m, n}+\frac{1}{2} \boldsymbol{W}_{a c}\left(\delta_{m-1, n}+\delta_{m+1, n}\right) .
$$

In this formalism, the time-reversal invariance amounts to $\hat{G}$ and $\hat{\Gamma}_{L, R}^{(k)}$ being symmetric, i.e. $\quad \hat{A}^{T}=\hat{A}$. Equation (B1) can then be proved by using the relations $[\hat{G}]_{m+k, n+k}(E)=[\hat{G}]_{m, n}(E+k \hbar \omega)$ and $\left[\hat{\Gamma}_{X}^{(l)}\right]_{m+k, n+k}(E)=\left[\hat{\Gamma}_{X}^{(l-k)}\right]_{m, n}(E+k \hbar \omega)$. We note that $\hat{\Gamma}_{X}^{(l)}$ is defined with a different sign of $l$ than in Ref. 4. 
* Electronic address: janne.viljas@kit.edu

1 S. J. van der Molen, H. van der Vegte, T. Kudernac, I. Amin, B. L. Feringa, and B. J. van Wees, Nanotechnology 17, 310 (2006).

2 D. C. Guhr, D. Rettinger, J. Boneberg, A. Erbe, P. Leiderer, and E. Scheer, Phys. Rev. Lett. 99, 086801 (2007).

3 S. Kohler, J. Lehmann, and P. Hänggi, Phys. Rep. 406, 379 (2005).

4 J. K. Viljas and J. C. Cuevas, Phys. Rev. B 75, 075406 (2007).

5 J. K. Viljas, F. Pauly, and J. C. Cuevas, Phys. Rev. B 76, 033403 (2007).

6 J. Buker and G. Kirczenow, Phys. Rev. B 66, 245306 (2002).

7 A. Tikhonov, R. D. Coalson, and Y. Dahnovsky, J. Chem. Phys. 116, 10909 (2003).

8 A. Tikhonov, R. D. Coalson, and Y. Dahnovsky, J. Chem. Phys. 117, 567 (2002).

9 M. Galperin and A. Nitzan, Phys. Rev. Lett 95, 206802 (2005).

10 I. Urdaneta, A. Keller, O. Atabek, and V. Mujica, Int. J. Quant. Chem. 99, 460 (2003).

11 I. Urdaneta, A. Keller, O. Atabek, and V. Mujica, J. Phys. B: At. Mol. Opt. Phys. 38, 3779 (2005).

12 E. R. Bittner, S. Karabunarliev, and A. Ye, J. Chem. Phys. 122, 034707 (2005)

13 S. Welack, M. Schreiber, and U. Kleinekathöfer, J. Chem. Phys. 124, 044712 (2006).

14 C. Liu, J. Speyer, I. V. Ovchinnikov, and D. Neuhauser, J. Chem. Phys. 126, 024705 (2007).

15 G.-Q. Li, M. Schreiber, and U. Kleinekathöfer, Europhys. Lett. 79, 27006 (2007).

16 J. Lehmann, S. Kohler, P. Hänggi, and A. Nitzan, Phys. Rev. Lett. 88, 228305 (2002).

17 M. Galperin and A. Nitzan, J. Chem. Phys. 124, 234709 (2006).

18 P. A. Orellana and M. Pacheco, Phys. Rev. B 75, 115427 (2007).

19 U. Harbola, J. B. Maddox, and S. Mukamel, Phys. Rev. B 73, 075211 (2006).

20 S. Kurth, G. Stefanucci, C.-O. Almbladh, A. Rubio, and E. K. U. Gross, Phys. Rev. B 72, 035308 (2005).

21 M. Galperin and S. Tretiak, arXiv:0712.1166.

22 H. M. McConnell, J. Chem. Phys. 35, 508 (1961).

23 V. Mujica, M. Kemp, and M. A. Ratner, J. Chem. Phys. 101, 6849 (1994).

24 V. Mujica, M. Kemp, A. Roitberg, and M. Ratner, J. Chem. Phys. 104, 7296 (1996).

25 A. Nitzan, Annu. Rev. Phys. Chem. 52, 681 (2001).

26 D. Segal, A. Nitzan, and P. Hänggi, J. Chem. Phys. 119, 6840 (2003).

27 Y. Asai and H. Fukuyama, Phys. Rev. B 72, 085431 (2005).

28 D. Segal, Phys. Rev. B 72, 165426 (2005).

29 A. Painelli, Phys. Rev. B 74, 155305 (2006).

30 S. K. Maiti, Chem. Phys. 221, 254 (2007).

31 J. K. Tomfohr and O. F. Sankey, Phys. Rev. B 65, 245105 (2002).

${ }^{32}$ V. Mujica, M. Kemp, and M. A. Ratner, J. Chem. Phys. 101, 6856 (1994)

${ }^{33}$ M. P. Samanta, W. Tian, S. Datta, J. I. Henderson, and C. P. Kubiak, Phys. Rev. B 53, R7626 (1996).
${ }^{34}$ H. Dalgleish and G. Kirczenow, Phys. Rev. B 73, 245431 (2006).

35 C. A. Stafford, D. M. Cardamone, and S. Mazumdar, Nanotechnology 18, 424014 (2007).

${ }^{36}$ F. Pauly, J. K. Viljas, and J. C. Cuevas, arXiv:0709.3588.

37 P. K. Tien and J. P. Gordon, Phys. Rev. 129, 647 (1963).

38 A. H. Dayem and R. J. Martin, Phys. Rev. Lett. 8, 246 (1962).

39 In general $\tau(E, V)$ will also depend on $T=\left(T_{L}+T_{R}\right) / 2$ and $\Delta T=T_{L}-T_{R}$. However, we will either consider $\Delta T=0$, or assume a linear-response regime with respect to $\Delta T$ and $\Delta \mu$ such that the dependence on $\Delta T$ does not play a role. Furthermore, we concetrate on the limit of low temperatures and will thus neglect the dependence on $T$ as well.

40 H. B. Akkerman and B. de Boer, J. Phys.: Condens. Matter 20, 013001 (2008).

41 M. Paulsson and S. Datta, Phys. Rev. B 67, 241403(R) (2003).

42 P. Reddy, S.-Y. Jang, R. A. Segalman, and A. Majumdar, Science 315, 1568 (2007).

43 A.-P. Jauho, N. S. Wingreen, and Y. Meir, Phys. Rev. B 50, 5528 (1994).

44 M. Wagner and W. Zwerger, Phys. Rev. B 55, R10217 (1997).

45 S. Grafström, J. Appl. Phys. 91, 1717 (2002).

46 G. Platero and R. Aguado, Phys. Rep. 395, 1 (2004).

47 The electron spin is not considered explicitly in the basis. It only appears as the factor 2 in $G_{0}$ and the expressions for electrical current.

48 S. Datta, Electronic transport in mesoscopic systems (Cambridge, Cambridge, 1995).

49 T. Markussen, R. Rurali, M. Brandbyge, and A.-P. Jauho, Phys. Rev. B 74, 245313 (2006).

50 T. N. Todorov, Phys. Rev. B 54, 5801 (1996).

51 J. K. Viljas, J. C. Cuevas, F. Pauly, and M. Häfner, Phys. Rev. B 72, 245415 (2005).

52 The sign of both hopping integrals should actually be negative to reproduce the correct order of eigenstates, such that the lowest-energy one has no "nodes". Here we consider $\gamma$ and $\eta$ to be positive, such that the actual hoppings are $-\gamma$ and $-\eta$. This differs from Ref. 36 .

${ }^{53}$ F. Pauly, J. K. Viljas, J. C. Cuevas, and G. Schön, arXiv:0705.3285.

54 L. Venkataraman, J. E. Klare, M. S. Hybertsen, and M. L. Steigerwald, Nature 442, 904 (2006).

55 L. Venkataraman, Y. S. Park, A. C. Whalley, C. Nuckolss, M. S. Hybetrsen, and M. L. Steigerwald, Nano Lett. 7, 502 (2007).

56 M. H. Pedersen and M. Büttiker, Phys. Rev. B 58, 12993 (1998).

57 J. R. Tucker and M. J. Feldman, Rev. Mod. Phys. 57, 1055 (1985).

58 U. Sivan and Y. Imry, Phys. Rev. B 33, 551 (1986).

59 X. Zheng, W. Zahng, Y. Wei, Z. Zeng, and J. Wang, J. Chem. Phys. 121, 8537 (2004).

60 M. Elbing, R. Ochs, M. Koentopp, M. Fischer, C. von Hänisch, F. Weigend, F. Evers, H. B. Weber, and M. Mayor, Proc. Natl. Acad. Sci. 102, 8815 (2005).

61 M. Tinkham, Introduction to Superconductivity, 2nd ed. (McGraw-Hill, New York, 1996).

62 F. J. Kaiser, P. Hänggi, and S. Kohler, Eur. Phys. J. B 54, 
201 (2006).

63 D. Segal and A. Nitzan, Chem. Phys. 281, 235 (2002).

${ }^{64}$ K. S. Thygesen and A. Rubio, J. Chem. Phys. 126, 091101 (2007).

65 Y. Dahnovsky, V. G. Zakrzewski, A. Kletsov, and J. V.
Ortiz, J. Chem. Phys. 123, 184711 (2005)

66 S. Datta and M. P. Anantram, Phys. Rev. B 45, 13761 (1992).

67 J. H. Shirley, Phys. Rev. 138, B979 (1965). 